\title{
Prolapso de la cúpula vaginal. Revisión del manejo médico y quirúrgico
}

\author{
Janer Sepúlveda Agudelo, Eliana Maribel Quintero, Martín Bernardo Mejía, Simón Oróstegui Correa*; Miguel Angel \\ Alarcón Nivia**
}

\section{RESUMEN}

El prolapso de cúpula vaginal no es causa de mortalidad pero si afecta la calidad de vida. Suele estar acompañado de enterocele y en ocasiones de rectocele y/o cistocele con una incidencia entre el 0.5 y el $2 \%$.

Siempre que nos enfrentemos a una paciente con prolapso de cúpula vaginal debemos buscar métodos que mejoren la calidad de vida y que preserven en lo posible la función sexual.

El tratamiento va desde el uso de pesarios hasta procedimientos quirúrgicos ya sea por vía vaginal o por vía abdominal.

Los objetivos del manejo quirúrgico son el alivio de los síntomas, la corrección del defecto del piso pélvico, la restauración del eje normal de la vagina, de la función vesical normal e intestinal y el mantenimiento de un potencial sexual satisfactorio.

El abordaje abdominal causa menos dispareunia, menor recurrencia y no afecta el ángulo vaginal; la vía vaginal consume menos tiempo con recuperación postquirúrgica más rápida y menor posibilidad de lesión intestinal.

En el presente artículo se hace una revisión del manejo médico y quirúrgico de prolapso de cúpula vaginal.

PALABRAS CLAVES: Prolapso de cúpula vaginal, pesarios, colpopexia sacroespinosa, colposacropexia.

\section{SUMMARY}

Although vaginal vault prolapse isn't a cause of mortality, it affects the quality of life. Frecuently it goes with enterocele, cystocele and rectocele, has been estimadet to ocurr in 0.5 and $2 \%$.

The goals of medical or surgical management are relief of symptoms, correction of all pelvic floor defects, restoration of the normal vaginal axis, restoration of normal bowel and bladder function and maintenance of the potentialy satisfactory sexual intercourse.

Abdominal surgery cause less dyspareunia. Vaginal surgery has less morbidity, hospital stay and intestinal injury.

This is review of the medical and surgical management of vaginal vault prolapse.

KEY WORDS: Vaginal vault prolapse, pessary, sacrospinous colpopexy, sacral colpopexy.

\section{Introducción}

Cerca del $14 \%$ de la población es mayor de 65 años, y se calcula que este porcentaje aumentará hasta casi un $20 \%$ en el año 2.000 o poco después.

A medida que aumenta la expectativa de vida entre las mujeres, la eversión masiva de la vagina es uno de los problemas que los ginecólogos observan con mayor fre-

\footnotetext{
* Médicos Residentes II Ginecología y Obstetricia. Hospital Universitario Ramón González Valencia. Universidad Industrial de Santander. Bucaramanga.

** Profesor Departamento de Ginecología y Obstetricia. Hospital Universitario Ramón González. Valencia. Universidad Industrial de Santander. Bucaramanga. Fellow Epidemiología Clínica.
}

cuencia. Si bien este trastorno no disminuye la supervivencia ni representa una amenaza de muerte para la paciente, afecta de manera significativa su calidad de vida, produce gran malestar al sentarse y al caminar, interfiere el coito normal y con la función vesical lo cual predispone a infección urinaria recurrente. Este problema es muy importante para el ginecólogo clínico, y constituye todo un reto por ser un trastorno complejo. Las pacientes asintomáticas no deben recibir ningún tratamiento; en quienes tienen síntomas, se debe seleccionar el tratamiento más adecuado que brinde el máximo beneficio y el menor riesgo.

Por lo tanto se debe estar familiarizado con las dos grandes vías de acceso, abdominal y vaginal, para corregir este problema. 


\section{Definición y clasificación}

El prolapso de la cúpula es la eversión masiva de la vagina, que generalmente está acompañado de enterocele y puede o no estar acompañado de rectocele y/o cistocele (1-2).

Se clasifica en tres grados:

Grado I: No llega al introito.

Grado II: llega al introito

Grado III Va más allá del introito.

\section{Epidemiología}

La incidencia de prolapso vaginal consecutivo a cualquier tipo de histerectomía va del 0.5 al $2 \%$ (3). Es más frecuente en mujeres blancas que en las negras (1).

\section{Mecanismos de soporte vaginal}

Los músculos que dan soporte a la vagina son:

a. Diafragma pélvico: Formado por el músculo elevador del ano y el coccígeo (o isquicoccígeo).

b. Diafragma urogenital formado por el músculo transverso profundo del periné y la fascia profunda del periné (o aponeurosis perineal media). El músculo coccígeo se origina en la cara interna de la espina ciática y en el borde anterior de la escotadura ciática mayor. Termina en la cara y borde anterior de las dos últimas vértebras sacras y en las dos vértebras coccígeas. Está inervado por ramos del plexo sacro (4).

El músculo elevador de ano está compuesto por dos fascículos: El pubococcígeo y el ilio-coccígeo.

El pubococcígeo tiene dos fascículos, el músculo pubovaginal y el pubo-rectal. El pubococcígeo se origina en la cara interna del pubis hasta el canal obturador; insertándose en el ligamento ano-coccígeo (4).

El fascículo ilio-coccígeo se origina en la cara interna de la espina ciática y en el arco tendinoso del elevador del ano. Termina en el ligamento ano-coccígeo y en el hueso cóccix

La membrana perineal fija la vagina y el cuerpo perineal a la entrada de la pelvis ósea, sostiene el piso pélvico contra los efectos del aumento de presión intraabdominal, y contra la gravedad

\section{Mecanismos que favorecen el prolapso de cúpula}

\section{a. Ocasionados por la histerectomia}

- Se cortan y se dejan con o sin soporte los ligamentos redondos y anchos.

- Se cortan y se dejan con o sin soporte los ligamentos uterosacros y cardinales.

La pérdida de estos soportes relajan el diafragma pélvico y la membrana perineal.

\section{b. Factores de riesgo prequirúrgico}

- Debilidades de la integridad de los tejidos de la paciente.

- Trauma obstétrico.

- Obesidad.

- Aumento de la presión intraabdominal (tos crónica).

- Alteraciones de la inervación en esta zona.

- Congénita.

- Iatrogénica $(2,5)$ c. Factores post quirúrgicos:

- Infección.

- Hematoma.

\section{Síntomas del prolapso}

- Protrusión del tejido o "bulto" a través del introito.

- Sensación de pujo.

- Sensación de cuerpo extraño genital

- Dolor dorsal.

- Incontinencia urinaria de esfuerzo.

- Ocasionalmente hemorragia.

\section{Tipos de prolapso de cúpula vaginal}

-Prolapso vaginal simple o puro, que suele ocurrir sólo o con enterocele hasta un $85-90 \%$ (2).

-Prolapso vaginal completo o compuesto que tiene un cistocele o un rectocele o ambos.

\section{Manejo médico}

En épocas antiguas se utilizaron múltiples tratamientos que se pensaban eficaces e incluían masajes y el uso de diversas sustancias químicas, astringentes y emolientes, para producir contracción de la musculatura longitudinal de la vagina.

Posteriormente, se prescribieron ejercicios gimnásticos. En uno de ellos se pedía a una paciente que colocara una tabla de $50 \mathrm{~cm}$ de largo entre las rodillas y después contrajera los músculos aductores, para ayudar a la retracción de la masa protuyente hacia la pelvis.

Algunas pacientes eran suspendidas de los tobillos durante períodos prolongados. Después, se ataron cabos a este aparato de suspensión, de los cuales se tiraba alternativamente, con la intención de "sacudir" la tumoración dentro de su posición normal (este método llamado técnica de "sucusión hipocrática", llevó a la técnica actual de sucusión abdominal, para detectar la presencia de ascitis intraperitoneal). En otro tratamiento, se acercaba a la masa protuyente una aguja o hierro candente, pues se pensaba que el temor del contacto haría que la paciente contrajera los músculos longitudinales de la pared vaginal y redujera el prolapso. Con el paso del tiempo, se introdujeron varias formas de pesarios para sostén, como recursos no quirúrgicos, a fin de mantener la masa protuyente dentro de la pelvis. Actualmente se usan modificaciones de estos últimos métodos.

\section{Medidas generales}

- Cambios en el estilo de vida por ejemplo dejar de levantar objetos pesados.

- Reposición estrogénica ya sea por vía sistémica o local. Esto se asocia con un incremento de la elasticidad tisular con la recuperación del espesor vaginal normal y con un aumento de la irrigación sanguinea.

- Tratamiento de los problemas médicos concomitantes: Cardiovasculares, constipación, trastornos respiratorios crónicos, alteraciones del metabolismo de los hidratos de carbono.

- Manejo de la obesidad. 
- Ejercicios de resistencia perineal que consisten en un programa regular de contracción voluntaria de los músculos del diafragma pélvico y del sistema esfinteriano de la pelvis. Mediante contracciones isométricas e estos músculos se fortalece el grupo de músculos perineales.

Un programa efectivo de ejercicios consiste en 15 contracciones voluntarias intensas cada una de tres segundos de duración seis veces por día.

\section{Pesarios}

Hay una variedad de indicaciones para usar pesarios en la atención de la paciente ginecológica (2).

El pesario no sólo es un recurso útil en vez de la intervención quirúrgica; también puede usarse como medida correctiva temporal hasta que pueda darse un tratamiento permanente. El pesario puede usarse durante mucho tiempo en pacientes con problemas médicos que impiden la intervención quirúrgica o que simplemente prefieren no operarse. La paciente debe entender que al menos al principio se requerirá revisiones ginecológicas cada dos o tres meses, para asegurarse de que el pesario no produce irritación local u otros problemas y hacerse citologías anuales. La paciente deberá recibir instrucción acerca de como retirar el pesario y la indicación de hacerlo cada 7 a 10 días para limpiarlo.

Nunca debe usarse en pacientes con enfermedad pélvica inflamatoria o vaginitis.

Los pesarios modernos están hechos de silicona que es flexible y fácil de moldear, de manera que las pacientes puedan colocárselos sin dificultad. No absorben secreciones vaginales y por tanto son fáciles de limpiar y tienen muy poco olor.

Hay cinco tipos de pesarios (2).

- Redondo o anular plegable: se comprime en una clipse y se introduce al recuperar su forma circular y se gira un cuarto de vuelta para que no se salga.

- Tipo rosquilla se introduce y se retira igual que el redondo.

- Inflable: Está hecho de caucho de látex y de diversos tamaños. Es una cámara inflable.

- Cúbico: Se comprime entre los dedos pulgar e indice y se introduce fácilmente en la vagina. Tiene seis superficies cóncavas que cuando se adosan la mucosa vaginal húmeda producen aspiración. Esto crea una presión negativa cuando el pesario recmplaza al aire en la vagina.

- El de Gelhorn: este pesario está fabricado de un material plástico de color claro, o silicona flexible con forma de hongo. El tallo presenta a veces un orificio que permite el drenaje vaginal y a veces lleva fijo un cordoncillo para facilitar la extracción.

\section{Manejo quirúrgico}

Medidas quirúrgicas preventivas de prolapso de cúpula vaginal cuando se realiza histerectomía vaginal:

Se considera que el prolapso de cúpula vaginal es prevenible al realizar una cuidadosa culdoplastia al tiempo de la histerectomía vaginal (1). Se describen a continuación varias técnicas.
- Técnica de culdoplastia posterior de Mc Call descrita en 1956 que suspende el fondo de saco vaginal posterior a los ligamentos uterosacros y cierra el fondo de saco de Douglas aproximando los ligamentos uterosacros en la línea media $(1-2,6-8)$ se utilizan 1 a 3 suturas de polidioxanona No 2-0. Según el grado de prolapso del fondo de saco posterior.

- Fijación profiláctica de la cúpula al ligamento sacroespinoso durante la histerectomía vaginal sobre todo cuando las estructuras de soporte (ligamentos uterosacros y cardinales) aparecen elongados y atróficos.

Algunos autores defienden la fijación profiláctica de la cúpula vaginal el ligamento sacroespinoso después de la histerectomía (Dony (9), Richardson (10) y Cruikshank (11). Otros investigadores (Morley (3) y Meeks (12) afirman que dada la baja frecuencia de prolapso de cúpula post histerectomía es una medida innecesaria y que debe reservarse como medida terapéutica para cuando esta complicación se ha producido. Se recomienda su realización sólo cuando se cumplen los siguientes criterios (13).

a. Elongación y atrofia total del complejo de ligamentos útero sacro y cardinales.

b. Severa laxitud de las estructuras de soporte del piso pélvico, que permiten que la cúpula vaginal protuya hasta más allá del introito después de las histerectomias y las plastias.

c. Prolapso total del útero en el momento de la histerectomía.

Medidas quirúrgicas preventivas de prolapso de cúpula vaginal cuando se realiza histerectomía abdominal:

Técnica de Moschowitz (1-2,6) se basa en el uso de una sutura no absorbible $2: 0$ en jareta o en bolsa de tabaco, comenzando en la parte mas inferior del fondo de saco de Douglas y tomando la precaución de incluir cualquier resto de ligamento úterosacro, la vagina posterior, sólo peritoneo en la parte lateral y sólo mordidas superficiales de serosa sobre el colon sigmoide.

Técnica de Halban, en lugar de utilizar una sutura en bolsa de tabaco como en el Moschowitz, el saco de Douglas se cierra con suturas sagitales. Se realizan 5 ó 6 suturas superficiales con material no reabsorbible o de reabsorción tardía 2:0 a través del peritoneo de la pelvis anterior y posterior, incluyendo la cara posterior de la vagina y la pared anterior del recto (14).

\section{Objetivos del manejo quirúrgico}

- Alivio de los síntomas.

- Corrección del defecto del piso pélvico.

- Restauración del eje normal de la vagina.

- Restauración de la función vesical normal e intestinal.

- Mantenimiento potencial sexual satisfactorio (1, 12).

Existen tres técnicas quirúrgicas:

- Método reconstructivo por vía vaginal. Abordaje por vía abdominal

- Procedimicnto obliterante: colpocleisis

\section{Métodos reconstructivos por vía vaginal}

\section{Revisión histórica}

Paul Swifel, en Alemania fue probablemente el primer médico que intentó una solución quirúrgica de este 
problema (3). El describió en 1892 una técnica de fijación sacrotuberosa para la suspensión de la cúpula vaginal.

En 1909 White corrigió cistoceles por vía vaginal anclando la vagina en la línea blanca del arco tendinoso de la fascia pélvica visceral. En 1927 Miller describió un método trasvaginal para corregir la eversión de la cúpula vaginal utilizando los ligamentos sacrouterinos para suspenderla a la cara anterior del sacro.

Amreich publicó en 1951 su fijación vaginal sacrotuberosa, técnicamente similar a la descrita por Sweifel. Fue resucitada por Sederl en 1958, quien utilizó la fijación sacrotuberal vaginal y la sacrotuberal coccígea o sacra llamadas Amreich II y I respectivamente (15). En 1963, Byron Inmon describió la fijación bilateral de la cúpula vaginal a la fascia de los músculos ileococcígeos en tres casos. Richter reintrodujo en 1967 la fijación sacrotuberosa en Europa y un año después describió el uso del ligamento sacroespinoso para mejorar su técnica (15). Randall y Nicholls introdujeron la técnica de fijación sacroespinosa en Estados Unidos hace apenas 20 años (3).

Colpopexia sacroespinosa transvaginal $(1-2,6,13-$ 14, 16).

- En posición de litotomía. Se toma la vagina prolapsada en cuatro cuadrantes con pinza de Allis.

- Se hace una incisión clíptica circundante en el prolapso de la cúpula vaginal, después de calcular la longitud de la vagina para reconstruir.

- Se hace disección roma u cortante y se separa del saco del enterocele alejándola del mismo y de la pared rectal anterior.

- Se localiza el ligamento sacro espinoso del lado derecho que tiene forma triangular con una trayectoria desde la espina ilíaca hasta el borde ipsilateral del sacro. Esté ligamento tiene 7 a $8 \mathrm{~cm}$ de longitud y está rodeado por el músculo coccigeo. (Ver fig.1)

- Se coloca una pinza de Allis en el ligamento. Se colocan 3 ó 4 puntos de material absorbible o permanente sintético según la preferencia a través del ligamento, no alrededor de él, para cvitar los vasos glúteos inferiores y 2 a $3 \mathrm{~cm}$ hacia la línea media de la espina ciática para evitar los vasos u nervios pudendos.

Siempre ha habido controversia en cuanto a qué suturas utilizar si absorbible o permanente. La mayoría de autores sostienen que las suturas permanente son mejores porque conceden un soporte duradero (17-19) pero por otro lado las suturas reabsorbibles por la fibrosis local que produce proveen fijación a largo plazo $(3,12)$. Las molestias ocasionadas por compresión nerviosa cuando se utilizan suturas reabsorbibles disminuyen a medida que va desapareciendo el material (20). Y finalmente las suturas no absorbibles están mas propensas a desgarro en especial cuando se utiliza polipropileno (13).

Se cierra el saco del enterocele. El exceso de peritoneo y mucosa vaginal redundante se extirpa. Se colocan puntos de Mc Call. El nuevo vértice vaginal se cierra con surget. Las suturas colocadas en el ligamento sacro espinoso se unen al nuevo vértice vaginal.

Implementos especiales para sujetar el ligamento sacro espinoso:

A través de los años se han ideado implementos para sujetar el ligamento sacroespinoso, entre ellos están:

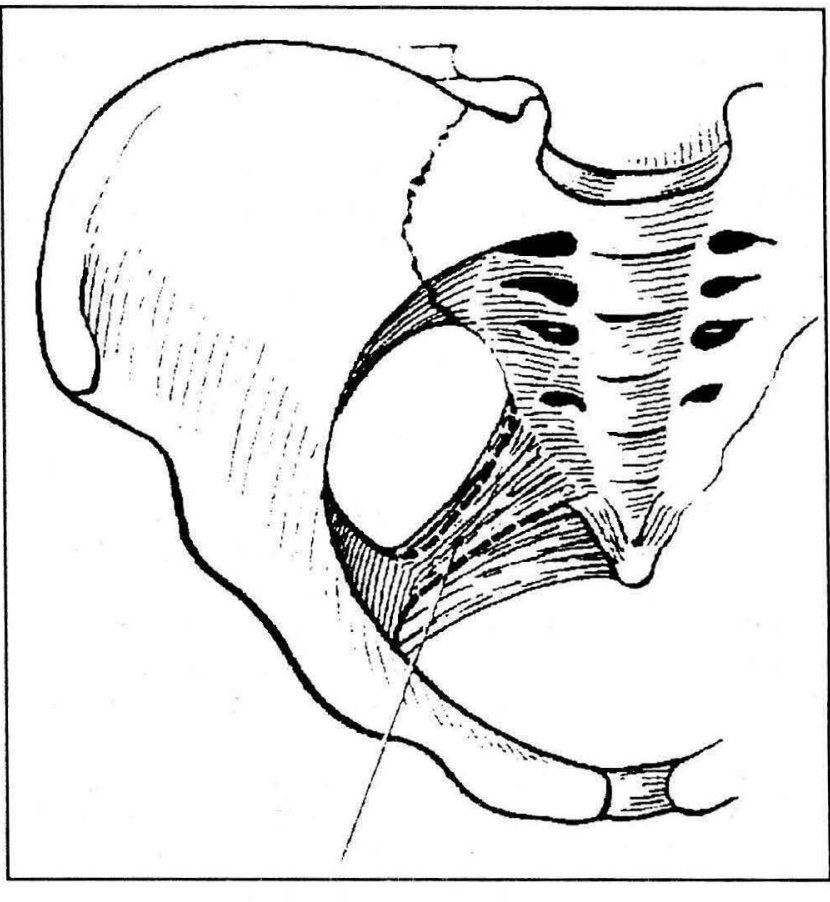

Figura 0006i01

COLPOPEXIA SACROESPINOSA

Ligamentos sacroespinosos y músculo coccígeo.

Anatomía de los ligamentos sacroespinosos, los cuales están tapados por el músculo coccígeo.

El porta sururas de Des Champs, el gancho de Miya y el porta agujas Haeney $(1,21)$.

Schelesinger (22) utilizó un mecanismo de autosutura endostitch de $36 \mathrm{~cm}$ de largo, instrumento utilizado para cirugía endoscópica y logró una disminución del tiempo operativa, menor sangrado y menos estancia hospitalaria.

\section{Complicaciones}

Hemorragia excesiva (vasos hemorroidales medios, pudendos y las arterias y venas glúteas inferiores), atrapamiento del nervio ciático y pudendo, retención urinaria, inestabilidad del detrusor, morbilidad febril como en cualquier procedimiento postoperatorio (neumonia, infección de vias urinarias, abscesos). Laceración vesical o rectal, desviación del canal vaginal de su sitio y acortamiento vaginal (23).

\section{Ventajas: (24)}

Menor estancia hospitalaria, menor intervención quirúrgica se evita una incisión abdominal y se puede lograr la reparación directa de un cistocele o un rectocele en el mismo momento sin cambiar de posición.

\section{Desventajas (24)}

Cirugía de alta complejidad, mayor dispareunia, mayor riesgo de recidiva del prolapso; campo quirúrgico deficiente, mayor riesgo de lesión o atrapamiento neural y sangrado profuso en caso de lesión vascular. 


\section{Recurrencia:}

Se ha reportado entre el 2 y $4 \%$ (25). Se logra una curación completa entre el $88-90 \%(2,5)$

\section{Modificaciones a la técnica quirúrgica}

Pohland Frattarelli (26) proponen que en vez de realizar fijación al ligamento sacroespinoso solo del lado derecho se debe realizar de una forma bilateral aduciendo que hay mayor simetría del canal vaginal, se crea un punto de soporte bilateral, mayor amplitud vaginal y menos probabilidad de recurrencia de prolapso vesical y uretral. Esta es una experiencia preliminar con 66 pacientes (26) por lo cual se deben esperar resultados a largo plazo.

Rodney y cols (12) y Shull y cols (27) proponen fijación a la fascia ileococcígea bilateral. Esta es fácil de localizar y ocasiona menos retroversión de la vagina por lo tanto menos relajación de la pared vaginal anterior y menos probabilidad de prolapso vesical y uretral, menor hemorragia, compresión nerviosa y mayor amplitud vaginal.

Thomas (28) propuso en pacientes con prolapso de cúpula recurrente (dos o más fallas reparativas) y en los que presentan defectos de soporte vaginal anterior, la utilización de una malla de marlex colocada en parte anterior a nivel de la unión uretrovesical; en la parte posterior en el apex vaginal y lateralmente en la unión del obturador y la fascia de los elevadores con buenos resultados. En una serie de 24 pacientes ( 12 control y 12 en tratamiento), no tuvo ninguna recurrencia y como principal complicación un flujo anormal por la presencia de cuerpo extraño o la formación de granulación que causa manchado pero de fácil tratamiento con nitrato.

\section{Métodos reconstructivos por vía abdominal}

\section{Revisión histórica}

Colposuspensión Abdominal.

- Técnica de Ward: En 1938 se usaba aponeurosis de buey para suspender el vértice vaginal a la aponeurosis del recto anterior (29).

- Técnica de Ridley: Usaba dos bandas de lascia lata, llevadas retroperitonealmente para suspender la cúpula de la aponcurosis del recto anterior (29).

- Técnica de Beecham: En 1973 se usaba una pieza de fascia lata para suspender la cúpula a los rectos anteriores (29).

Colposuspensión al ligamento de Cooper, descrita por Langmade en 1965, realizaba desinserción bilateral del Cooper y unión a la cúpula a través de túneles bajo los redondos (29). Richardson y Williams en 1969 describieron una operación similar en la que se introducían tiras de aponeurosis del oblicuo externo a través de túneles paralelos a los ligamentos redondos y se usaban para suspender la cúpula vaginal (29). Ridley en 1976 describió una operación compuesta en la que se aseguraban bandas de fascia lata a la cúpula por vía vaginal, se pasaban retroperitonealmente al lado de los ligamentos redondos y se injertaban en la aponeurosis de los rectos abdominales anteriores (29).

En términos generales estas técnicas quirúrgicas producen un desplazamiento anormal de la vagina a un eje antifisiológico, que produce compresión vesical y disfunción sexual (29).

\section{Colpo-sacropexia técnica actual:}

\section{Materiales a usar:}

- Materiales de origen natural: Aponeurosis abdominal, fascia lata, duramadre; (usualmente son tejidos débiles dada la edad promedio de las pacientes).

- Materiales sintéticos: Mallas de Marlex, Dacrón, Látex, Goretex, Teflón.

- Suturas no absorbibles: Preferiblemente monofilamentos (29).

\section{Técnica quirúrgica}

Preparación prequirúrgica: Se recomienda llevar la paciente al acto quirúrgico con preparación mecánica del recto, y restricción de dieta durante las 24 horas previas (30).

La paciente es colocada en posición de litotomía con estribos universales de Allen. El lavado quirúrgico va desde el abdomen hasta el tercio superior de los muslos, incluyendo la cavidad vaginal; posteriormente se procede a instalar un catéter de Foley vesical (30).

Se recomienda el ingreso a la cavidad abdominal por línea media infraumbilical, ya que brinda una exposición óptima de la pelvis y la región anterior del sacro, además de brindar la posibilidad de extensión de la incisión en los casos necesarios. Pero la incisión de Pfannenstiel es una buena alternativa. Luego del ingreso a la cavidad abdominal se coloca un separador de Sullivan O'Connor o uno de Balfour. Se procede a realizar la adherenciolisis requerida, y posteriormente se procede a realzar y exponer la cúpula prolapsada para facilitar su fijación con el método que haya escogido el cirujano. Para la identificación de la cúpula puede ser útil un molde, un dilatador de Hegar o simplemente los dedos (29-30).

Se procede a colocar de 9 a 12 puntos de suturas no absorbibles, separados, de manera circunferencial alrededor de la cúpula prolapsada, que incluyan la pared vaginal en todo su espesor (30).

Se recomiendan suturas de grosor y tensión adecuadas (calibre 0 ) además de no absorbibles.

Luego, se procede a pasar las suturas previamente colocadas, a través de una capa sencilla de la malla (cortada en forma cónica) alrededor de cúpula prolapsada, y se fijan dichas suturas.

Se retrae el colon sigmoide hacia la izquierda, y se incide el peritoneo posterior en la línea media, un poco por arriba del promontorio, y se extiende la incisión hasta S3-S4. Se realiza una dirección cuidadosa del tejido perisacro evitando-lesionar la vasculatura de dicha región, que puede producir hemorragias de muy difícil control con las medidas tradicionales.

Una vez lograda la exposición, se procede a colocar de 3 a 5 suturas permanentes, interrumpidas, en el periostio de la cara anterior del sacro en los niveles S3-S4, cuidando de no lesionar los plexos vasculares y los agujeros sacros anteriores (31).

A continuación, se procede a realizar la obliteración del fondo de saco de Douglas mediante una culdoplastia de Halban o de Moschowitz. 
Se procede luego a fijar la malla en la cara anterior del sacro, evitando la tensión excesiva. Se pasan a través de la malla los puntos previamente colocadas a nivel del sacro, se anudan y se cortan. Se cierra la hoja posterior del peritoneo, cubriendo completamente la malla (31). (Ver fig 2)

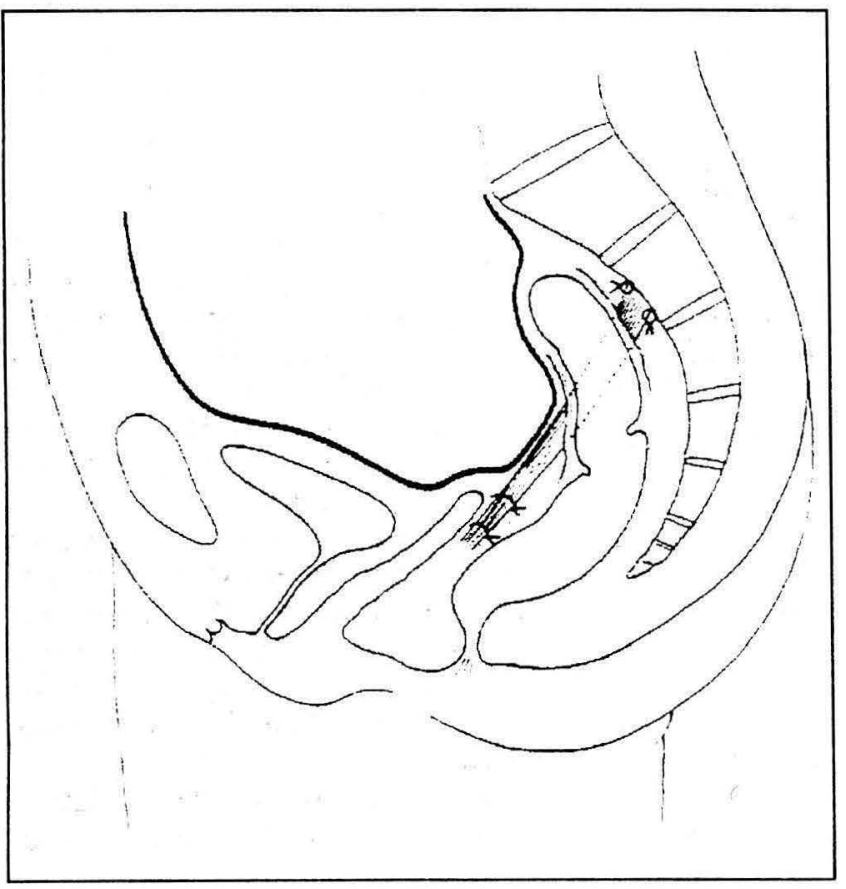

Figura $0006 \mathrm{i} 02$

SACROCOLPOPEXIA

Orientación fisiológica del eje de la vagina

\section{Manejo de la pared vaginal anterior}

Si previo al acto quirúrgico se ha diagnosticado incontinencia urinaria de esfuerzo se debe realizar una colposuspensión de Burch (29).

\section{Manejo de la pared vaginal posterior}

Se recomienda evaluar el rectocele hasta después de finalizada la colposacropexia, ya que se considera que al finalizar esta se puede restablecer el sostén normal y eliminar el rectocele.

En caso de no desaparecer se justificaría realizar un colporrafía posterior.

\section{Complicaciones post quirúrgicas (32-34)}

1. Erosión de la malla a través de la cúpula.

2. Retención urinaria

3. Infección del tracto urinario

4. Abscesos retroperitoneales.

5. Tromboflebitis.

Es de anotar que dichas complicaciones, se presentan en muy contadas ocasiones y son factibles de ser prevenidas.

\section{Técnicas alternativas de Colpo-sacropexia}

1. Suspensión con clavo de titanio al sacro.

Consiste en la introducción de un clavo de titanio en el promontorio, donde es fijada la malla que eleva la cúpula vaginal. Su propósito es evitar la posibilidad de lesión vascular presacra. Se trata de una técnica experimental (35).

\section{Colpoperineopexia}

El descenso perineal excesivo se presenta como consecuencia de una variedad de desórdenes ano-rectales que incluyen: Constipación, incontinencia fecal, dolor rectal, síndrome de úlcera rectal solitaria, rectocele y enterocele. Además el descenso perineal anormal se ha relacionado con el desarrollo posterior de incontinencia fecal ocasionada por neuropatía pudenda por atrapamiento y elongación del nervio. Las reparaciones quirúrgicas del rectocele y enterocele no corrigen el descenso perineal, por lo cual la neuropraxia y denervación del piso pélvico y el esfínter anal persisten, ocasionando no solo incontinencia fecal, sino también predisponiendo a prolapso de cúpula recurrente por miopatía por denervación (36).

En esta técnica el espacio recto-vaginal es disecado hacia abajo hasta el aspecto superior de la fascia posterior de la vagina, contigua con el cuerpo perineal. Si se encuentran separadas las fascias vaginales anterior y posterior, se reaproximan con suturas permanentes.

Se suturan piezas trapezoidales de malla a los aspectos anteriores y posterior de la vagina.

$\mathrm{Si}$ la fascia posterior se encuentra rota o muy adelgazada, la malla posterior se sutura directamente al cuerpo del periné.

Se suturan las piezas de la malla a la pared vaginal posterior con una sutura no absorbible manofilamentosa, y posteriormente se sigue la técnica ya descrita.

Es de anotar que por lo reciente de la técnica, no se han realizado evaluaciones confiables (36).

3. Colporrectosacropexia

No es común que se presenten simultáneamente prolapsos genitales y rectales en una misma paciente.

En esta técnica el recto es llevado hacia la izquierda, se incide el peritoneo posterior a la línea media a nivel del promontorio del sacro en la unión del recto y sigmoide. Se identifica el uréter derecho. Se diseca el espacio retrorectal hasta el coxis. Se corrige el prolapso rectal. Se sutura una malla en forma trapezoidal de $12 \mathrm{cms}$ de longitud a la pared posterior del recto, a nivel de la ampolla rectal, con una sutura no absorbible, de tal manera que la malla cubra la pared posterior del recto. El otro extremo de la malla se lleva a nivel de S2-S3 en el sacro. Se corta otra porción de malla, que se debe unir al ligamento intervertebral S2-S3 del sacro y a la pared lateral derecha del recto, de tal manera que lo fije a la izquierda del sacro y la porción distal de la malla es suturada a la pared posterior y superior de la vagina (37). (Ver fig 3 )

\section{Ventajas de la colposacropexia Abdominal}

Dispareunia ocasional. Menor riesgo de recurrencia del prolapso. Buen campo quirúrgico. Recupera el ángulo vaginal normal. Ofrecen posibilidad de corrección de cistocele retropúbico en el mismo acto quirúrgico (24). 


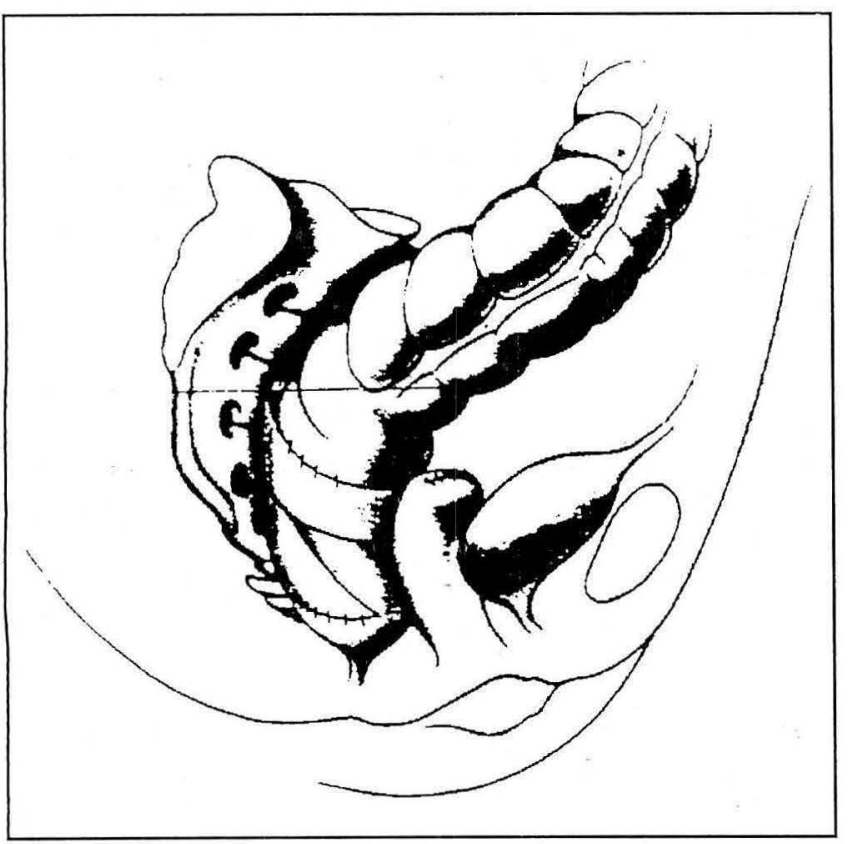

Figura 0006i03

COLPORECTOSACROPEXIA

Corrección simultánea del prolapso genital y rectal. Visión esquemática.

\section{Desventajas}

Mayor riesgo de lesiones vesicales e intestinales. Mayor tiempo quirúrgico y por ende mayores costos hospitalarios. Mayor dolor post quirúrgico (24).

\section{Técnicas Obliterativas: Colpocleisis}

Sólo es aplicable a pacientes mayores, que no desean ser sexualmente activas en el futuro y presentan un riesgo quirúrgico alto.

\section{Técnicas quirúrgicas}

Se realiza una incisión circular en la base de la vagina, que compromete la mucosa. Ayudado por el dedo del cirujano se realizan la denudación de la mucosa vaginal hasta la interfase mucoso-facial. A medida que se retira la mucosa vaginal se va realizando hemostasia selectiva.

Se procede a realizar afrontamiento de la fascia anterior y posterior de la vagina con sutura absorbible, reduciendo el cele a medida que se avanza, hasta llegar al introito vaginal.

El procedimiento finaliza con el cierre de la mucosa vaginal superior a la posterior a nivel del introito (38).

La otra técnica descrita para colpocleisis total o parcial es la técnica de Le Fort, la cual consiste en la resección de colgajo trapezoidales de mucosa en las caras anterior y posterior de la vagina; con posterior atrapamiento de las mismas con sutura absorbible, cuidando de dejar libres los ángulos laterales bilaterales, que servirán de drenaje de las secreciones vaginales.

\section{Otras alternativas quirúrgicas}

Colpopexia Video- Laparoscópica:

En el duodécimo Congreso Mundial de Ginecología y Obstetricia, Pinotti y Palma mostraron una nueva técnica quirúrgica para el tratamiento del prolapso de cúpula vaginal en el cual la vagina prolapsada es llevada hacia la pared abdominal anterior y fijada a la fascia de los músculos rectos con un abordaje abdomino perineal con control endoscópico (39).

Aún tiene alto porcentaje de recidivas. Requiere mayor experiencia.

\section{Agradecimientos}

A los doctores Oscar Gómez León, Enrique Rueda Pinilla y Claudia Parra Barón; y en especial a la Dra. Lucy Elena Sanguino Abril por la colaboración en la revisión del tema.

\section{BIBLIOGRAFIA}

1. Nichols D.H. Central compartment defects: Enterocele and massive eversion of the vagina. In: Thompson J, Rock JA. Te Linde Is operative gynecology. Eight edition Philadelphia. Edit Lippincott Raven 1997; 1003-1030.

2. Morley GW. Tratamiento para la eversión de la cúpula vaginal a través de la vía vaginal. In: Clínicas Obstétricas y Ginecológicas. México: Interamericana Mc Graw Hill. 1993; 4: 926-933.

3. Morley, George W., De Lancey J. Sacrospinous ligament fixation for eversion of the vagina. American Journal of Obstetrics and Gynecology 1988; 158: 872-881.

4. De lancey JO. Anatomía y biomecánica del prolapso genital. In: Clínicas Obstétricas y Ginecológicas. México: Interamericana Mc Graw Hill. 1993; 4: 845-855.

5. Magazine. Diagnosing and manogiry genitourinary prolapse. British Medical Journal. March 221997.

6. Lee RA. Atlas de Cirugía ginecológica. México. Interamericana McGraw Hill. 1995; 76-92.

7. Hirsch HA. Uterosacral ligament suspension of vagynal vault (Mc Call's culdoplasty). Europa Journal. Obstetrice Gynecology Reproduction Biology 1989; 32(1): 13.

8. Given FT. Posterior culdoplasty revisted. American Journal ObstetricsGynecology 1985; 153: 135-139.
9. Dony IM. Treatment of vagynal vault prolapse. Neth Journal Surgery 1989; 14(6): 152-155.

10. Richadson DA., Scotti R.J., Ostergard DR. Surgical management of uterine prolapse in young women. J Reprod Med 1989; 34: 388-392.

11. Cruikshank SH., Cox D. Sacrospinous ligament fixation at the time transvagynal hysterectomy. American Journal of Obstetrics and Gynecology 1990; 162: 1611-1619.

12. Meeks RG., Washburne JF., McGehee RP., Wiser WL. Repair of vaginal vault prolapse by suspension of the vagina to iliococcygeus (prespinous) fascia. Am J Obstet Gynecol 1994; 1444-1454.

13. Osorio Orozco O., Cifuentes CA. Colpopexia Sacroespinosa. Rerista Medicina de Caldas 1993; 13(1): 21-26.

14. Hirsch HA., Kaser O., Iklé FA. Atlas de cirugía ginecológica. Madrid, España. Marban 1997; 116-121. 279-284.

15. Richter Kurt. Massive eversion of the vagina: pathogenesis, diagnosis and therapy of the "true" prolapse of the vagynal stump. Clinical Obstetrics and Gynecology 1982; 25: 897-912.

16. Verdeja AM., Elkins TE., Odoi A., Gaser R., Lamoutte C. Transvaginal sacrospinous colpopexy: Anatomic landmarks to be aware of to minimize complications. Am J Obstet Gynecol 1995; 173: $1468-1469$ 
17. Thornton WN., Peters WA III. Repair of vaginal prolapse after hysterectomy. Am J Obstet Gynecol 1983; 147: 140-8.

18. Shull BL., Capen CV., Riggs MW., Kuehl TJ. Bilateral attachment of the vaginal cuff to iliococcygeus fascia: an effective method of cuff suspension. Am J Obstet Gynecol 1993; 168: 1669-1677.

19. Kethel ML., Heberson R. Anatomic Evaluation of the sacrospinous ligament colpopexy. Surgery Gynecology and Obstetrics 1989; 168 : 318-322.

20. Bayron MR., Cortázar PA., Celades JF. Colpopexia sacrospinosa: una alternativa razonable para el manejo del prolapso de cúpula vaginal por vía vaginal. Revista Colombiana de Obstetricia y Ginecología 1996; 47: 241-245.

21. Miyazaki FS. Miya Hook ligature carrier for sacrospinous ligamente suspension. Obstetrics and Gynecology 1987; 70: 286.

22. Schlesinger RE. Vaginal sacrospinous ligament fixation with the Autosuture endostitch device. Am J Obstet Gynecol 1997; 176: 13581362.

23. Alevizon SJ., Finan MA. Sacrospinous colpopexy: Management of postoperative pudendal nerve entrapment. Obstet and Gynecol 1996; 88: 713-715.

24. Benson JT., Lucente V., Mc Clellan E. Vaginal Vs Abdominal recontructive surgery for the treatment of pelvic support defects: $A$ prospective condomited study with long term outcome evaluation. Am J Obstet Gynecol 1996: 175: 1418-1422.

25. Hardiman PJ., Drutz HP. Sacrospinous vault suspension and abdominal colposacropexy: Success rates and complications. Am J Obstet Gynecol 1996; 175: 612-616.

26. Pohl JF., Frattarelli JL. Bilateral transvaginal sacrospinous colpopexy: Preliminary experience. Am J Obstet Gynecol 1997; 177: 1356-1362.

27. Shull BL., Capen CV .. Riggs MW., Kuehl T.J. Bilateral attachment of the vaginal cuff to iliococygeus fascia: An affective method of cuff suspension. Am J Obstet Gynecol 1993; 168: 1669-1677.

28. Thomas MJ. The efficacy of Marlex mesh in the repair of severe, recurrent vaginal prolapse of the anterior midvaginal wall. Am J Obstet Gynecol 1996; 175: 1472-5
29. Addison WA., Timmons MC. Tratamiento para la eversión de la cúpula vaginal a través de la vía abdominal In: Clínicas Obstétricas y ginecológicas. México: Interamericana Mc Graw Hill. 1993; 4: 935-944.

30. Addison WA., Timmons MC. Abdominal sacral colpopexy for the treatment of vaginal vault prolapse with enterocele. In: Thompson J. Rock JA. Te Linde Is operative gynecology. Eight edition Philadelphia. Edit Lippincott Raven 1997; 1030-1036.

31. Hendee AE. Sacral colpopexy for enterocele and vaginal vault prolapse. In: Thompson J. Rock JA. Te Linde. Is operative gynecology. Eight edition Philadelphia. Edit Lippincott Raven 1997; 1037-1044.

32. Drutz. HP., Cha LS. Massive genital and vaginal vault prolapse treated by abdominal-vaginal sacropexy with use of Marlex mesh: Review of the literature. Am J Obstet Gynecol 1987; 156(2): 387-392.

33. Snyder TE., Krantz. KE. Abdominal-retroperitoneal sacral colpopexy for the correction of vaginal prolapse. Obstet Gynecol 1.991; 77 : 944-949.

34. Grunberger W., Grunberger V., Wierrani F. Pelvic promontory fixation of the vaginal vault in sixty-two patients with prolapse after histerectomy. Journal of the american College of Surgeons 1994; 178: 69-72.

35. Smith MR. Colposacropexy: An alternative technique. Am J Obstet Gynecol 1997; 176: 1374-1375.

36. Cundiff GW., Harris RL., Coates K., Low Vincent HS. Bump RC. Addison WA. Abdominal Sacral Colpoperineopexy: A new approach for correction of posterior compartment defects and perineal descent associated with vaginal vault prolapse. Am J Obstet Gynecol. 1997; 177: 1345-1353.

37. Tancer ML.. Fleischer M., Berkowitz J. Simultaneous colpo-rectoSacropexy. Obstetrics and Gynecology 1987; 70: 951-955.

38. De Lancey JO., Morley GW. Total colpocleisis for vaginal eversion. Am .J Obstet Gynecol 1997; 176: 1228-1235.

39. Palma PC.. Pinotti JA. Endoscopic suspension of vagynal prolapse. International Journal gynecology Obstetrics 1988; $27(3)$ : 451-454. 